\title{
Photoreflectance characterization of InAs/GaAs self-assembled quantum dots grown by ALMBE
}

\author{
M. Geddo ${ }^{1,2, a}$, R. Ferrini ${ }^{3}$, G. Guizzetti ${ }^{3}$, M. Patrini $^{3}$, S. Franchi ${ }^{4}$, P. Frigeri ${ }^{4}$, G. Salviati ${ }^{4}$, and L. Lazzarini $^{4}$ \\ 1 INFM-UdR Pavia, Via Bassi 6, 27100 Pavia, Italy \\ 2 Dipartimento di Fisica dell'Università di Parma, Viale delle Scienze 7a, 43010 Fontanini (Parma), Italy \\ 3 INFM - Dipartimento di Fisica "A. Volta", Università di Pavia, Via Bassi 6, 27100 Pavia, Italy \\ ${ }^{4}$ CNR-MASPEC Institute, Parco delle Scienze 37a, 43010 Fontanini (Parma), Italy
}

Received 17 November 1999

\begin{abstract}
We report on a photoreflectance investigation in the $0.8-1.5 \mathrm{eV}$ photon energy range and at temperatures from 80 to $300 \mathrm{~K}$ on stacked layers of InAs/GaAs self-assembled quantum dots (QDs) grown by Atomic-Layer Molecular Beam Epitaxy. We observed clear and well-resolved structures, which we attribute to the optical response of different QD families. The dependence of the ground state transition energy on the number of stacked QD layers is investigated and discussed considering vertical coupling between dots of the same column. It is shown that Coulomb interaction can account for the observed optical response of QD families with different morphology coexisting in the same sample.
\end{abstract}

PACS. 78.66.Fd III-V semiconductors - 73.20.Dx Electron states in low-dimensional structures (superlattices, quantum well structures and multilayers) - 78.40.-q Absorption and reflection spectra: visible and ultraviolet

\section{Introduction}

In the last few years increasing effort has been directed to the research of nanometer scale devices like quantum dot (QD)-based lasers, whose specific lasing characteristics promise to compete strongly with the quantum well (QW)-based ones. Although the optical properties of InAs QDs have been extensively investigated by low temperature photoluminescence (PL) techniques [1], very few papers exist on QDs using modulation spectroscopy. Of these we mention the paper by Aigouy et al. [2] (contactless electroreflectance on 10-layer stacked QDs), that by Rowland et al. [3] (photoreflectance (PR) and PL on 2-layer stacked QDs) and the paper by Sun et al. [4] (photovoltage and $\mathrm{PR}$ of single layer QDs).

In this paper we present a PR investigation at different temperatures and for different numbers of QD layers of the optical transitions in InAs/GaAs stacked QDs grown by Atomic Layer Molecular Beam Epitaxy (ALMBE). Recently, it has been shown $[5,6]$ that ALMBE is particularly suited to growing relatively large self-assembled QDs, with relatively sharp size distribution and with emission wavelengths at room temperature located in the $1.3 \mu \mathrm{m}$ spectral window of photonic interest. On the other hand, their size dispersion and multimodal nature drastically limit the performances of QD-based devices.

\footnotetext{
a e-mail: geddo@fisav.unipv. it
}

The aim of this paper was to investigate the temperature dependence of the ground state transition energies associated to QD families with different morphology, as well as their dependence on the number of stacked QD layers.

\section{Experimental details}

The structures consisted of $100 \mathrm{~nm}$ thick GaAs buffer layers grown by $\mathrm{MBE}$ at $600{ }^{\circ} \mathrm{C}$ on (100) GaAs substrates, of $N=0$ to $N=9$ stacked pairs of layers of self-aggregated InAs QDs and of $10 \mathrm{~nm}$ thick overhanging GaAs spacer (or cap) layers. On top of the structure a single uncapped layer of QD may terminate the structure; the structures are described by the identifier $N / M$, where $N$ is the number of embedded QD layers, while $M$ is 0 or 1 depending on whether the uncapped topmost QD layer is absent or present, respectively. The QDs were grown by ALMBE at $460{ }^{\circ} \mathrm{C}$ and at $0.13 \mathrm{ML} / \mathrm{s}$, while the $10 \mathrm{~nm}$ thick spacer and caps were deposited by ALMBE at $360^{\circ} \mathrm{C}$. The InAs coverage was $3 \mathrm{ML}$. The growth technique (ALMBE) and the growth temperatures were chosen so as to optimize the PL properties of the structures.

Topographic images of uncapped dots were taken by contact-mode Atomic Force Microscopy (AFM) under constant probe force conditions. The measurements were performed using silicon nitride cantilevers and tips with 
curvature radii of $\sim 20 \mathrm{~nm}$. Then, the images were analysed in order to derive the base size and the height distribution of the dots, taking into account the convolution effects due to the finite dimension of the AFM stylus tip. TEM bright field and dark filed images of (110) crosssections were recorded with a JEOL 2000 FX microscope at $200 \mathrm{kV}$ on mechano-chemically thinned samples finished by argon ion-milling.

Ex-situ Atomic Force Microscopy (AFM) studies performed on uncapped structures show that the QD height $(h)$ and the diameter $(d)$ distributions have mean values that increase from $h=5 \mathrm{~nm}$ and $d=18 \mathrm{~nm}$ for single uncapped layer $(0 / 1)$ structures, to $h=7.3 \mathrm{~nm}$ and $d=38 \mathrm{~nm}$ for the topmost plane of QDs in $N / 1$ structures with $N=9[5,6]$. The QD-related $\mathrm{PL}$ peaks in capped structures have $10 \mathrm{~K}$ full widths at half maximum (FWHM) ranging from $50 \mathrm{meV}$ for $N=1$ to $22 \mathrm{meV}$ for $N=9$. Further details about growth conditions of the structures and about the ALMBE technique may be found in references $[5,6]$.

PR measurements were performed at near normal incidence in the $0.8-1.5 \mathrm{eV}$ range, with energy steps and spectral resolution of $1 \mathrm{meV}$. The standard experimental apparatus [7] operated with a $100 \mathrm{~W}$ halogen lamp as probe source. The excitation source was provided by a $2 \mathrm{~mW}$ He-Ne laser, mechanically chopped at $220 \mathrm{~Hz}$. The sample was mounted in thermal contact with the cold finger of a micro-miniature Joule-Thompson refrigerator, that allows measurements in the $80-300 \mathrm{~K}$ temperature range.

\section{Results and discussion}

PR signal was observed at different temperatures from all the relevant regions of the heterostructures, i.e. a) from the GaAs buffer layers, b) from the InAs wetting layers (WL), that uniformly cover the buffer layers and from which the QDs emerge, and c) from the InAs QDs. As an example, in Figure 1 we compare the $150 \mathrm{~K}$ PR spectra of structures with a single QD layer, with and without cap (i.e. $1 / 0$ and $0 / 1$ ). The high-energy part of the spectra of both samples is dominated by the PR feature associated to the fundamental band gap of the GaAs buffer. On the other hand, the spectral features we attribute to InAs QDs (QD1, QD2) show up only in the low-energy part of the spectrum of the capped structure. We assign these PR features to the optical response of QDs for two reasons: QD1 is very close (when corrected for the temperature shift) to the characteristic $10 \mathrm{~K} \mathrm{PL}$ emission energy of ALMBE QDs [5]; the absence of the QD features in uncapped structures is consistent with the presence of a "dead layer" at the sample surface. In fact, III-V surfaces are known to favour non-radiative recombination (related to the presence of surface states); this in turn usually causes the disappearance of exciton-related signals in PR (absorption) measurements. We noted that the intensity of these features $\left(\Delta R / R \sim 10^{-5}-10^{-6}\right)$ is slightly higher than that of recently reported PR signals attributed to the ground state transitions [3].

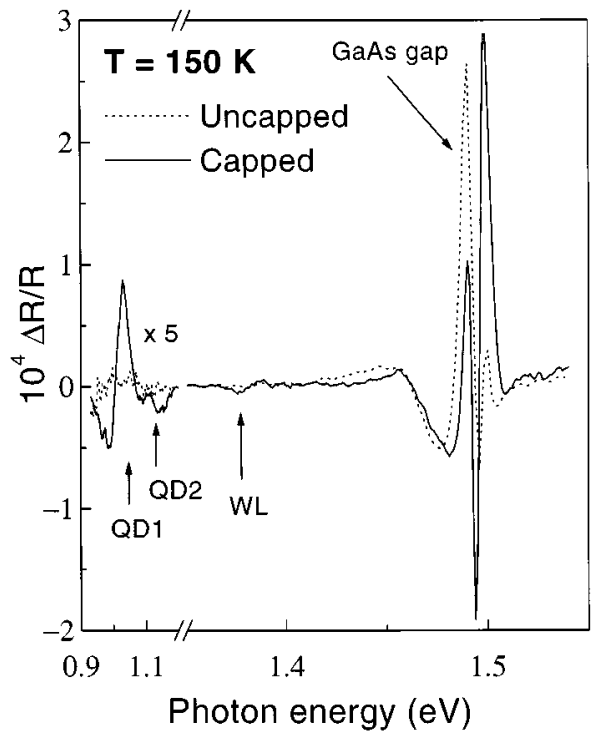

Fig. 1. Comparison of $150 \mathrm{~K} \mathrm{PR}$ spectra of structures with a single capped or uncapped QD layer. QD-related PR features are clearly evident in the capped structure near $1 \mathrm{eV}$. The weak QW-like PR feature near $1.39 \mathrm{eV}$ marks the optical response of the wetting layer.

At about $1.39 \mathrm{eV}$, just below the band gap of the GaAs buffer layer, the PR spectrum of the capped structure exhibits a weak and broadened QW-like feature attributable to the InAs WL. This feature was not detected in uncapped structures due to the aforementioned presence of a dead layer. Differently from what we previously observed in monolayer-sized InAs/GaAs single quantum wells [8], in the present case only the heavy-hole free-exciton PR feature could be detected, due to the weakness of the signal. The comparison with recently reported PL results $[9,10]$ concerning the relation between the critical thickness for QD nucleation and the optical response of WLs indicates an effective thickness of 1.6-1.8 ML, in agreement with the critical thickness for the 2D-3D transition of InAs grown on GaAs.

The optical spectra were analysed on the basis of the PR lineshape model characterising electromodulated signals from bound states, such as those in QD and QW heterostructures. Following Aigouy et al. [2], we used the first derivative of a Gaussian profile for the dielectric function; this profile is suitable for taking into account the inhomogeneous broadening related to size and thickness fluctuations of QDs and QWs, respectively. The Gaussian lineshape model we chose to interpret the PR optical response of QDs has already been successfully applied to the PR spectra of QWs [11], quantum wires [12] and, more recently, of stacked QDs [2,3] as well. We also checked different lineshape models like the band-to-band and the Lorentzian excitonic profiles with unsatisfactory results.

In Figure 2 we show the best-fit (solid line) of the QD $\mathrm{PR}$ features at $150 \mathrm{~K}$ (dotted line) of the $1 / 0$ structure, as obtained by using the first derivative of a Gaussian excitonic profile [11]. The fit quality was quite satisfactory, as indicated by a standard deviation of about $1 \times 10^{-6}$. 


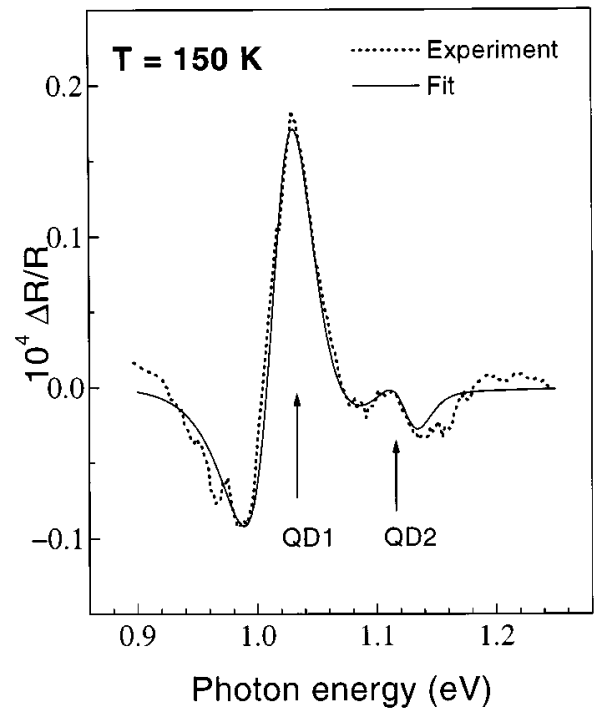

Fig. 2. Best-fit (solid line) of the $150 \mathrm{~K} \mathrm{PR}$ spectrum (dotted line) in the region of the QD optical response of a structure with a single capped QD layer (1/0). Arrows mark the ground state transition energies of the different QD families.

We obtained the values of $E_{1}=1.019 \mathrm{eV}$ and $\Gamma_{1}=$ $48 \mathrm{meV}$ for the transition energy and the broadening parameter of QD1 feature, while the corresponding values for the QD2 one were $E_{2}=1.126 \mathrm{eV}$ and $\Gamma_{2}=42 \mathrm{meV}$, respectively. Typical uncertainties in the $E$ and $\Gamma$ parameters were 2 and $4 \mathrm{meV}$, respectively. As will be observed later, the QD1 and QD2 features must be related to the ground state transitions of different QD families. Here we note that the broadening parameter values of the QD transitions $(40-50 \mathrm{meV})$ are much larger than those measured on III-V based QW structures (typically $3-5 \mathrm{meV}$ ) [11]: the different inhomogeneous broadenings are related to the different extent of the size dispersion (of the order of $7 \%$ or more for all families) in the case of self-aggregated QDs $[1,13]$, and of the thickness dispersion in the case of multi-QWs.

In Figure 3 we present the temperature dependence of the QD1 and QD2 transition energies (full circles and open circles, respectively) of the same $1 / 0$ structure. The first is shown to be intermediate between the temperature dependence of the InAs band gap [14] (solid line) and the GaAs one [15] (dotted line), which have been shifted in energy to facilitate the comparison. As shown in reference [1] the InAs and GaAs bandgaps are expected to be the limiting behaviour for large and small QDs.

As regards QD2, the second and less intense PR feature, due to its energy position (about $100 \mathrm{meV}$ higher than QD1), it should be attributed either to excited state transitions or to the ground state transition of a second QD family $[10,13,16]$, with a different size and/or morphology with respect to the main family. We lean towards the second attribution consistently with the observation of a different $N$ dependence for the QD1 and QD2 transition energies (see below) and with the morphological characterization by AFM and TEM.

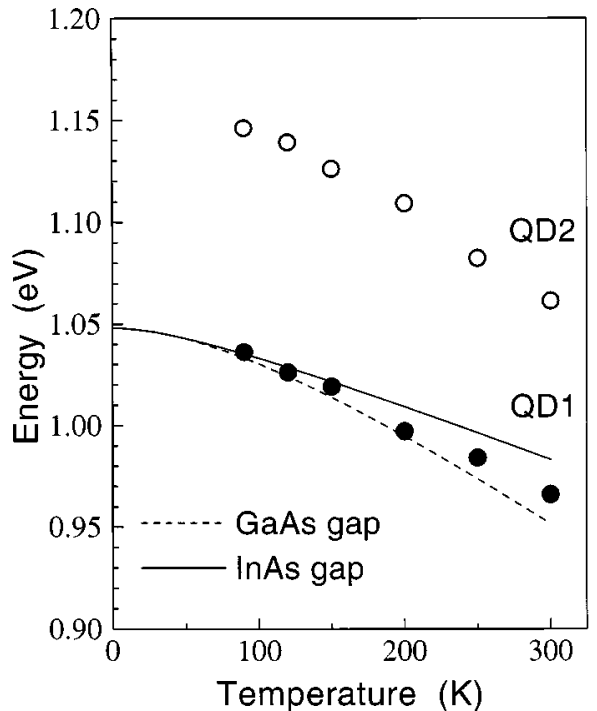

Fig. 3. Temperature dependence of the ground state transition energies for the main (full circles) and the second (open circles) dot families of a $1 / 0$ structure. The temperature dependence of the InAs (GaAs) fundamental band gap is given by the solid (dotted) line, shifted in energy to facilitate comparison.

Figure 4a shows an example of AFM image of structures with a single uncapped layer of QDs ( $0 / 1$ structures), while Figure 4b gives the base size distribution deduced from the images and obtained by the deconvolution of the profiles of 165 QDs on an area of $\left(0.35 \times 0.35 \mu \mathrm{m}^{2}\right)$. It is generally known that uncapped QDs may have morphologies and sizes somewhat different from that of the capped ones, due to the interaction among spacer and QDs [17]; however the base size distribution of QDs in uncapped structures (Fig. 4b) clearly indicates the existence of two QD families with base sizes of $15.5 \mathrm{~nm}$ and $18.5 \mathrm{~nm}$. Moreover, the height distribution (that is not affected by artefacts related to deconvolution) shows two peaks at $3.2 \mathrm{~nm}$ and $5.8 \mathrm{~nm}$. The smaller and larger QDs have aspect ratios $h / d$ of 0.21 and 0.31 , respectively. The base size values are influenced by the deconvolution procedure and, therefore, may not represent accurately the aspect ratio of the crystallographic facets, however they confirm the existence of two QD families with different sizes and facet indexes. Moreover, AFM results in structures with increasing $N$ suggest that the smaller QD family tends to disappear.

A cross-section observed by TEM of a $5 / 1$ structure (Fig. 5) further supports the existence of QD families with different sizes. It is interesting to observe that in all of the studied cross-sections, the smaller dots tend to disappear towards the top of the structure and that the smaller dots have higher facet indexes.

In reference [13] the emission energies of InAs/GaAs pyramidal QDs are calculated as a function of the base sizes and facet indexes resulting in higher energies for smaller dots and higher indexes. Extrapolating the data given in Table 3 of reference [13] to larger base sizes, we find that QD families with a base size ratio of 1.2 , as in our case, should emit at energies that differ by less than 

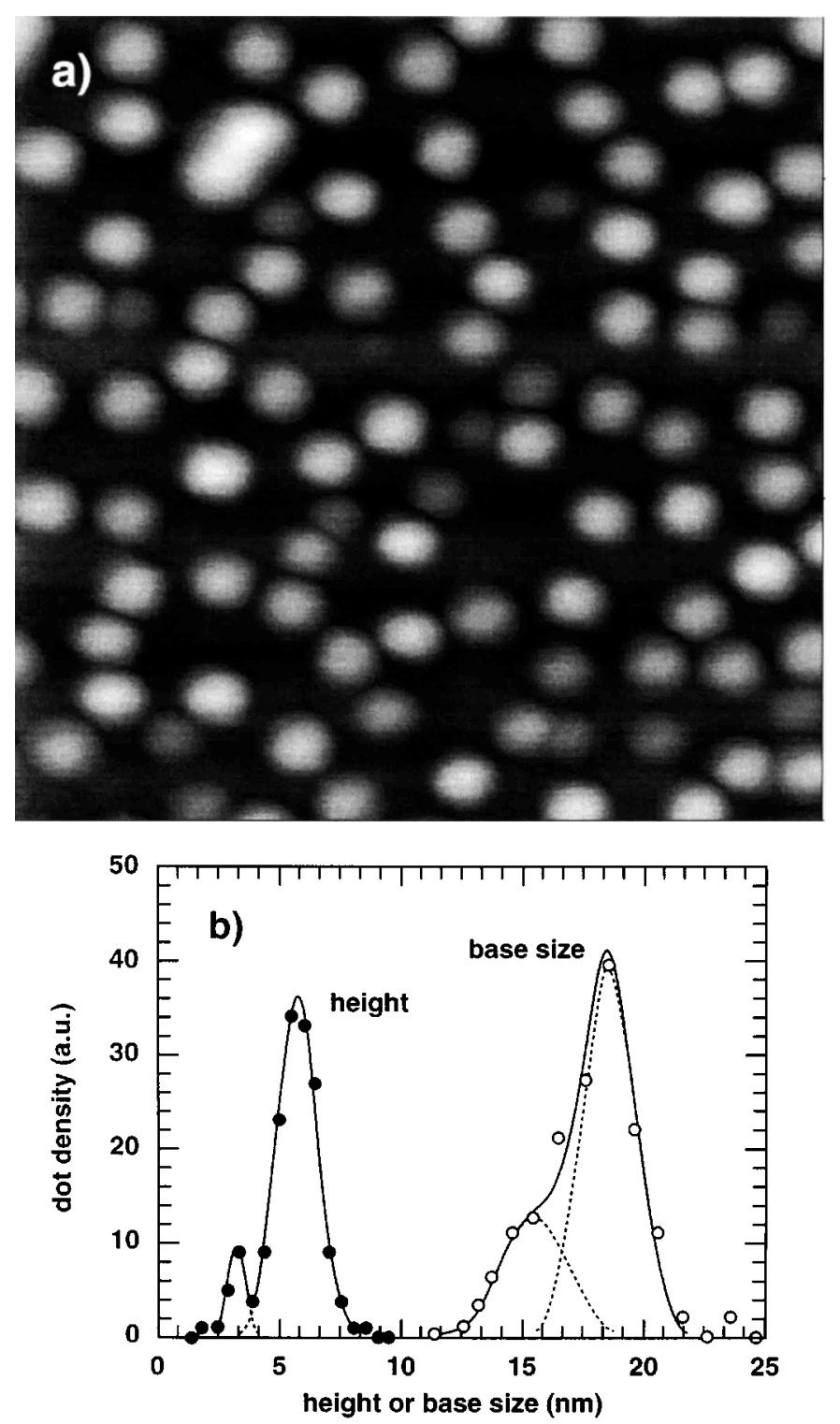

Fig. 4. $\left(0.25 \times 0.25 \mu \mathrm{m}^{2}\right)$ AFM micrograph of uncapped InAs QDs grown by ALMBE in a structure consisting of a single layer of QDs (a) and distribution of base sizes and heights of $\operatorname{dots}(b)$.

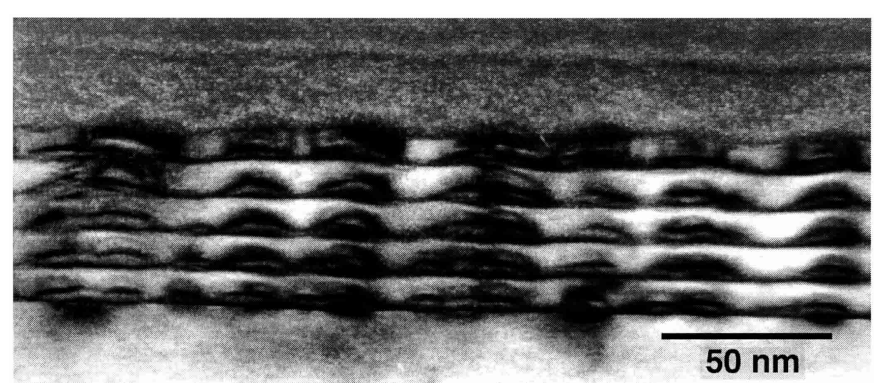

Fig. 5. (110) cross-sectional TEM image of a 5/1 structure taken under (002) dark field conditions which enhance the compositional contrast between InAs and GaAs, but are not suitable for imaging the topmost uncapped QD layer.

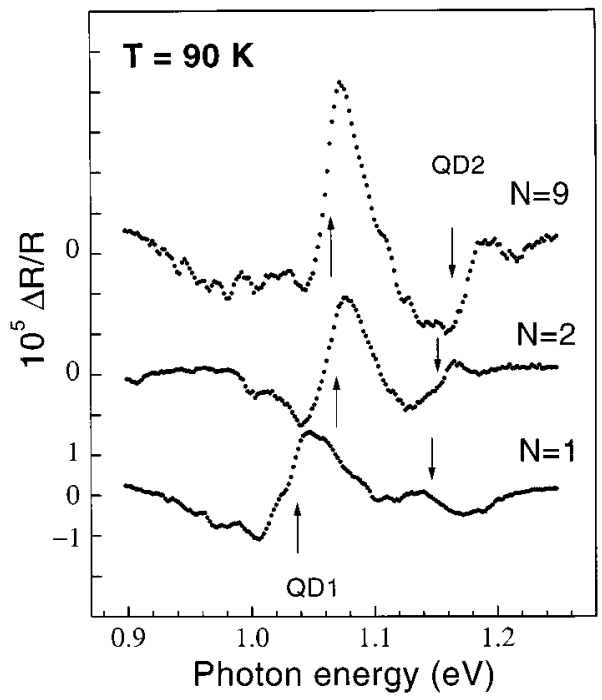

Fig. 6. Comparison between the $90 \mathrm{~K}$ PR spectra (vertically shifted for clarity) of samples with different number $N$ of embedded layers. Arrows mark the transition energies for the two QD families.

$50 \mathrm{meV}$; this difference increases to values close to the our experimental ones $(\sim 110 \mathrm{meV})$ if we consider that smaller dots have lower $h / d$ aspect ratios, i.e. higher facet indexes.

The $90 \mathrm{~K}$ PR spectra of the $1 / 0,2 / 1$ and $9 / 1$ structures are shown in Figure 6 (shifted vertically for clarity). The PR features we attribute to two different dots families are clearly evident in all spectra. Moreover, in the structure with single capped QD layer (1/0) structure a small shoulder appears on the QD1 feature, $\sim 50 \mathrm{meV}$ spaced in energy. According to reference [3] they can be attributed to excited state transitions and their weakness makes them hard to resolve in our samples. They are almost completely hidden by the increased intensity of the main features in the stacked sample spectra.

This is in agreement with reference [3], where it is shown experimentally that the intensity of the excitedstate features is one order of magnitude lower with respect to the ground-state ones. Moreover, it is well-known that in confined systems (e.g. in QWs [18]) the oscillator strengths of the excited states are much lower than those of the ground state transitions and that symmetry forbidden transitions $(\Delta n \neq 0)$ become allowed. According to recent theoretical results [19] this is true even in the case of QDs.

The relative intensity of the QD2-related spectral features decreases (by $\sim 20 \%$ ) as the number of embedded QD layers increases from $N=1$ to $N=9$ : this effect can be interpreted as due to an increase of the QD size uniformity, that is a general consequence of stacking QD layers [20].

Moreover, in Figure 6 we note that when the number of stacked layers is increased from 1 to 2 the main PR feature (QD1) displays a significant blue-shift $(\sim 30 \mathrm{meV})$. This is surprising if we consider that vertical ordering effects are expected to produce a progressive red-shift [21], due to vertical coupling of QDs belonging to the same 


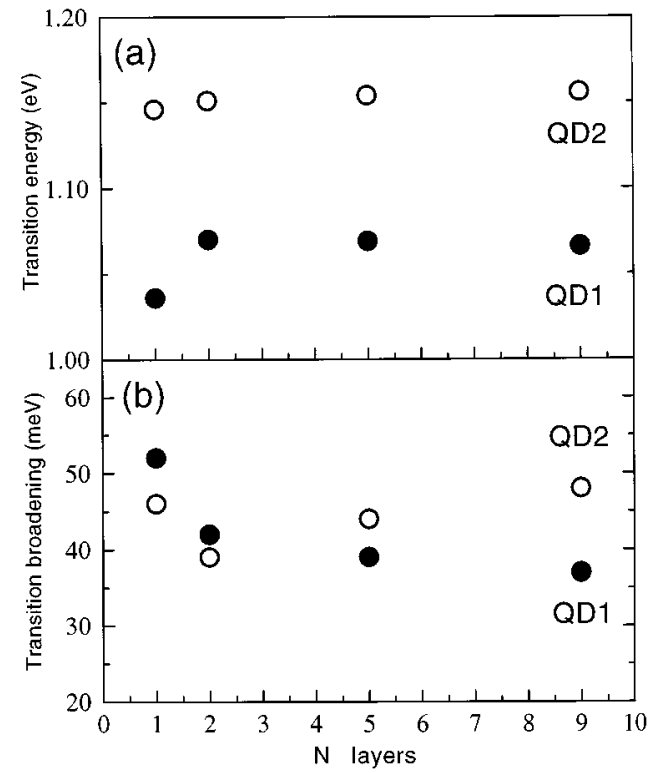

Fig. 7. $90 \mathrm{~K}$ experimentally derived transition energy (a) and broadening (b) as functions of the number of embedded layers for the QD1 (full circles) and QD2 (open circles) families.

column. A large red-shift has been observed [21] in QD structures where the QDs vertically interact more strongly than in the present case, due to the smaller spacer thickness $(6.2 \mathrm{~nm}$ instead of $10 \mathrm{~nm})$. Recently, it has been shown $[22,23]$ that Coulomb interaction between carriers inside the dot can explain this behavior: in particular, detailed calculations of electron and hole confining energies (performed on stacked arrays similar to our structures) versus the number of stacked QD layers indicate that the competing effects of Coulomb interaction and vertical coupling account for the observed drastic blue-shift when spacers $10 \mathrm{~nm}$ thick are used. Indeed, when a second layer is added, with $10 \mathrm{~nm}$ thick spacers, carrier wavefunction delocalization [23] produces a drastic decrease in the Coulomb interaction energy (blue-shift); successive layers favour the formation of minibands which, in turn, reduce (red-shift) the ground state transition energy (the QD "gap"). Vertical coupling (red-shift) does indeed become the dominant effect if spacers have the same size of QDs (i.e. $6 \mathrm{~nm}$ ) [21].

The experimentally derived transition energies and broadening parameters of the QD1 and QD2 families are reported in Figure 7. The abrupt blue-shift and the negligible blue-shift characterizing QD1 (full circles) and QD2 (open circles), respectively, show evidence of the different conditions of the two dot families. This behavior can be explained by reminding that the second QD family has a smaller aspect ratio, and then it sees thicker spacers between the QD planes. Therefore, the vertical coupling between QD2 dots is smaller than that characterizing the QD1 family; as a consequence, the QD2 dots tend to behave as not-interacting systems, with emission energies essentially independent of the number of stacked layers. This is also confirmed by the calculation in reference [23].
These results combined with the data reported in Figure $7 \mathrm{~b}$, showing the decrease of broadening parameter for the main PR feature (QD1) in stacked heterostructures, indicate for the ALMBE grown QDs the possibility of improving homogeneity and, at the same time, of getting monomodal distributions by using stacked QD arrays.

\section{Conclusions}

Systematic measurements at different temperatures were performed by $\mathrm{PR}$ on self-assembled InAs/GaAs QDs grown by ALMBE with a InAs coverage of $3 \mathrm{ML}$. In the spectral region of the characteristic emission energies of ALMBE dots $(\sim 1 \mathrm{eV})$ PR spectra have inferred the existence of two QD families with different size and aspect ratio. This observation is also supported by AFM and TEM results. The evolution of the ground state transition energy with the number of stacked layers has been related to the morphology of the QD family considered. The different behavior of the QD1 and QD2 family has been explained by the combined effects of Coulomb interaction and vertical coupling between QD of the same column. Moreover, a drastic improvement in performances (through greater ordering of the structures and monomodal distribution) of QD-based optoelectronic devices is expected if opportunely spaced stacked arrays are used.

This work was partially supported by the CNR-MADESS II Project and INFM Network "Fisica e tecnologia dei semiconduttori III-V". We have benefited from a number of helpful discussions with Prof. M. Capizzi, Prof. M. Colocci and Dr. S. Taddei, who are gratefully acknowledged.

\section{References}

1. For a recent review, D. Bimberg, M. Grundmann, N.N. Ledentsov, Quantum Dot Heterostructures (J. Wiley and Sons, London, 1999).

2. L. Aigouy, T. Holden, F.H. Pollak, N.N. Ledentsov, W.M. Ustinov, P.S. Kop'ev, D. Bimberg, Appl. Phys. Lett. 70, 3329 (1997).

3. G.L. Rowland, T.J.C. Hosea, S. Malik, D. Childs, R. Murray, Appl. Phys. Lett. 73, 3268 (1998).

4. B.Q. Sun, Z.D. Lu, D.S. Jiang, J.Q. Wu, Z.Y. Xu, Y.Q. Wang, J.N. Wang, W.K. Ge, Appl. Phys. Lett. 73, 2657 (1998).

5. A. Bosacchi, P. Frigeri, S. Franchi, P. Allegri, V. Avanzini, J. Cryst. Growth 175/176, 771 (1997).

6. A. Bosacchi, P. Frigeri, S. Franchi, P. Allegri, V. Avanzini, J. Cryst. Growth 201/202, 1136 (1999).

7. J.L. Shay, Phys. Rev. B 2, 803 (1970).

8. M. Geddo, M. Capizzi, A. Patanè, F. Martelli, J. Appl. Phys. 84, 3374 (1998).

9. M. Grassi Alessi, M. Capizzi, A.S. Bhatti, A. Frova, F. Martelli, P. Frigeri, A. Bosacchi, S. Franchi, Phys. Rev. B 59, 7620 (1999).

10. A.S. Bhatti, M. Grassi Alessi, M. Capizzi, P. Frigeri, S. Franchi, Phys. Rev. B 60, 2592 (1999). 
11. H. Shen, S.H. Panad, F.H. Pollack, Phys. Rev. B 37, 10919 (1988); J. Glembocki, B. Shanabrook, Semiconductors and Semimetals, edited by D.G. Seiler, C.L. Littler (Academic, Boston, 1992), Vol. 36, p. 221; F.H. Pollak, in Handbook on Semiconductors, edited by P. Balkansky (North-Holland, Amsterdam, 1994), Vol. 2, p. 527.

12. M. Geddo, S. Di Lernia, A. Stella, A. Bosacchi, S. Franchi, M. Gentili, D. Peschiaroli, Solid State Commun. 100, 221 (1996).

13. J. Kim, Lin-Wang Wang, A. Zunger, Phys. Rev. B 57, R9408 (1998).

14. Z.M. Fang, K.Y. Ma, D.H. Jaw, R.M. Cohen, G.B. Stringfellow, J. Appl. Phys. 67, 7034 (1990).

15. M. Guzzi, J.L. Staehli, Solid State Phen. 10, 25 (1989).

16. R. Heitz, A. Kalburge, Q. Xie, M. Grundmann, P. Chen, A. Hoffmann, A. Madhukar, D. Bimberg, Phys. Rev. B 57, 9050 (1998).
17. J.M. Garcia, G. Medeiros-Ribeiro, K. Schmidt, T. Ngo, J.L. Feng, A. Lorke, J. Kotthaus, P.M. Petroff, Appl. Phys. Lett. 71, 2014 (1997).

18. G. Bastard, Wave mechanics applied to semiconductor heterostructures (Les Éditions de Physique, Les Ulis, 1992).

19. L. Silvestri, G. Czajkowski, F. Bassani, Phys. Status Solidi A 175, 383 (1999).

20. J. Tersoff, C. Teichert, M.G. Lagally, Phys. Rev. Lett. 76, 675 (1996).

21. G.S. Solomon, J.A. Trezza, A.F. Marshall, J.S. Harris Jr., Phys. Rev. Lett. 76, 952 (1996).

22. M. Colocci, A. Vinattieri, L. Lippi, F. Bogani, M. Rosa-Clot, S. Taddei, A. Bosacchi, S. Franchi, P. Frigeri, Appl. Phys. Lett. 74, 564 (1999).

23. S. Taddei, M. Colocci, A. Vinattieri, F. Bogani, S. Franchi, P. Frigeri (private communication). 